\title{
Mitigating Collisions through Power-Hopping to Improve 802.11 Performance *
}

\author{
Paul Patras *, Hanghang Qi, David Malone \\ Hamilton Institute, National University of Ireland Maynooth, Ireland
}

\begin{abstract}
In this article, we introduce a power-hopping technique (PH-MAC) that, by alternating between different transmission power levels, aims to deliberately cause packet capture and thereby reduce the impact of collisions in 802.11 WLANs. We first devise an analytical model of the 802.11 protocol with heterogeneous capture probabilities, and show that, depending on the network load, the capture effect can enhance the throughput performance of all nodes. We base the design of PH-MAC on the findings following from this analysis and demonstrate that important performance improvements can be achieved by exploiting the interactions between the MAC and PHY layers to mitigate collisions. Finally, to understand the feasibility of this technique in practical deployments, we present a prototype implementation of PH-MAC which relies on commodity hardware and open-source drivers. We evaluate the performance of this implementation in an indoor testbed under different network conditions in terms of link qualities, network loads and traffic types. The experimental results obtained show that our scheme can provide significant gains over the default 802.11 mechanism in terms of throughput, fairness and delay.
\end{abstract}

Key words: 802.11, power-hopping, capture effect

\section{Introduction}

In practical IEEE 802.11 deployments, when two or more simultaneous transmissions occur on the channel, if frames arrive at the destination with different power

\footnotetext{
ऋ The research leading to these results has received funding from the European Community's $7^{\text {th }}$ Framework Programme (FP7-ICT-2009-5) under grant no. 257263 (FLAVIA project), the Irish HEA PRTLI Cycle 4 FutureComm and Science Foundation Ireland grant no. 07/SK/I1216a.

* Corresponding author. Address: NUI Maynooth, Co. Kildare, Ireland.
} 
levels, it is often the case that the one received with the strongest signal can be demodulated despite the interference caused by the others, provided the difference in their signal strength is sufficiently large. This phenomenon is referred to as the capture effect and has been widely neglected when modeling the IEEE 802.11 protocol behaviour, the common assumption being that simultaneous transmissions result in collisions [1-4].

Recent works show both analytically and experimentally that the capture effect can potentially reduce the number of failures due to collisions and thus increase the throughput performance of the network [5-11]. However, we argue that the interactions between the MAC and PHY layers in the presence of the capture effect have not been yet deeply understood, since existing studies neglect the impact of traffic load and postulate that only nodes that deliver frames at high signal levels (generally due to their better placement relative to the access point) benefit from capture.

In contrast to previous works, in this paper we first devise an analytical model of the 802.11 operation under the capture effect and show that this phenomenon can not only improve the overall throughput of the network, but may also enhance the performance of the nodes that deliver frames at a low signal level, when the stations experiencing better link qualities with the receiver are lightly loaded. Specifically, we show that, depending on the network load, the throughput attained by the stations residing further away from the access point (AP) is not always degraded due to nodes located near the AP capturing the channel, but, on the contrary, the capture effect can also reduce the collision rate encountered by the distant users, thereby providing them with larger throughput. Our model adopts a renewal-reward process [3] to model the binary exponential backoff scheme of the MAC protocol and extends our previous analysis of [12] by considering a fully heterogeneous network, i.e. distinguishing multiple classes of nodes that experience dissimilar capture probabilities.

Based on the valuable insights that follow this analysis, we introduce a powerhopping MAC/PHY scheme (PH-MAC) that exploits the identified protocol behaviour to boost the WLAN performance. Specifically, our proposal preserves the 802.11 MAC rules, but selects among different power levels with certain probabilities when transmitting frames, deliberately causing capture to mitigate collisions. This design extends our earlier work [12], which assumed stations could only choose among a high and a lower power level. Here, we allow for multiple discrete power levels and also investigate the impact of their number. We model this enhancement using a Bianchi-type Markov chain [1] and show that, by choosing the power levels with equal probabilities, PH-MAC reduces the impact of collisions, providing significantly better throughput performance as compared to the standard 802.11 protocol.

To evaluate the potential gains of our mechanism, we implement a practical approx- 
imation of PH-MAC using open-source drivers and off-the-shelf 802.11 hardware. We assess the performance of this prototype by conducting extensive experiments in a small-scale indoor testbed, under different conditions in terms of link qualities, network loads and traffic types. The obtained results show that PH-MAC can achieve noteworthy performance gains over the default 802.11 scheme in terms of total throughput, fairness and delay, without requiring any changes to the existing hardware, but only small modifications to the available device drivers.

The rest of the paper is organised as follows: in Section 2, we review relevant related work; in Section 3 we present the network model considered for our analysis; in Section 4 we undertake an analytical and numerical study of the 802.11 performance with heterogeneous capture; in Section 5, we present the power-hopping MAC scheme that we implement and validate both numerically and via experiments with real devices; finally, Section 6 concludes the paper.

\section{Related Work}

Aspects of the capture effect have been widely studied in the past in the context of mobile radio environments, e.g. $[13,14]$. Despite the significant effort devoted to modelling the performance of 802.11 DCF (see e.g. [1-4]), the capture effect is largely ignored in these studies, as well as in recent publications that investigate the behaviour of the EDCA protocol enhancement [15-17]. To date few analytical models of the capture probability in Rayleigh fading channels have been proposed and used to predict the impact of this effect on the capacity of 802.11 networks, e.g. [6,7]. Ge et al. [5] take a further step towards understanding how capture affects the back-off mechanism of the protocol, while Sutton et al. [8] introduce a more detailed 3-dimensional Markov chain model that incorporates capture and serves estimating not only failure probability, but also several QoS metrics. However, the failure probability is computed by subtracting a capture probability from the collision probability. Instead, in our analysis we treat a fraction of the collisions as resulting in capture.

Experimental studies have examined the capture phenomenon comprehensively with real deployments, identifying the throughput unfairness that may arise due to this effect $[9,18]$. A deeper understanding of how frame timing and signal strength influence the occurrence of capture in practice is provided in [10,11]. Based on the arrival time of a frame with a stronger signal relative to the arrival of weak signal packet, Manweiler et al. distinguish between capture and message-in-message phenomena, and exploit the latter to improve network throughput [19]. To the best of our knowledge, no previous approaches adjust the transmission power to purposely cause capture and benefit from this effect.

Transmit power control techniques have been employed to improve the energy- 
efficiency of the 802.11 DCF [20] or for mitigating interference in multi-AP deployments [21]. A combined rate and power control scheme is proposed in [22] to improve battery-life of mobile devices, while avoiding link asymmetries and improving capacity. These works, confirm the feasibility of dynamically adapting the transmission power with current 802.11 devices, which constitutes one the motivations behind the design of the power-hopping technique we propose herein for reducing the impact of collisions. However, as compared to these approaches, our objective is to better understand the PHY/ MAC interactions under the capture effect and exploit those in dense environments to enhance network performance.

\section{Network Model}

In this section, we provide an overview of the network model and the assumptions used in the performance analysis that we conduct. We consider the case of infrastructure 802.11 wireless networks, i.e. all transmissions are to/from the AP. We start by introducing relevant aspects of the IEEE 802.11 protocol with the DCF (Distributed Coordination Function) operation, which is the default channel access scheme currently employed in WLANs [23] and then explain how capture is accounted for in our system.

\subsection{IEEE 802.11 DCF}

DCF uses a CSMA/CA (Carrier Sense Multiple Access with Collision Avoidance) MAC protocol with binary slotted exponential backoff. Briefly, when a station having packets to send senses the wireless medium idle for a period of DIFS (Distributed Inter-frame Space), it initialises a backoff counter with a random value uniformly distributed in the $[0, C W]$ interval and enters a countdown state. Specifically, as long as the medium remains idle, the node decrements its backoff counter by one after each slot time $\sigma$, and suspends the countdown if the medium becomes busy. The countdown is resumed once the medium is idle again and when the backoff counter reaches zero, the station transmits. The $C W$ parameter is called the

Table 1

802.11b PHY/MAC parameters.

\begin{tabular}{lr|lr}
\hline \hline$C W_{\text {min }}$ & 31 & Slot time $(\sigma)$ & $20 \mu \mathrm{s}$ \\
\hline$m$ & 5 & Data rate & $11 \mathrm{Mb} / \mathrm{s}$ \\
\hline DIFS & $50 \mu \mathrm{s}$ & PLCP duration & $96 \mu \mathrm{s}$ \\
\hline SIFS & $10 \mu \mathrm{s}$ & ACK duration & $106 \mu \mathrm{s}$ \\
\hline \hline
\end{tabular}


contention window and its value is set to a default $C W_{\min }$ upon the first transmission attempt.

If a transmitted frame is successfully received, the destination sends back an acknowledgement (ACK) frame after a period of SIFS (Short Inter-frame Space) to notify the sender of the correct reception. In thr case where the backoff counters of two or more stations reach zero simultaneously, the stations transmit in the same time slot and thus a collision occurs. If the destination cannot decode a frame, no ACK is sent back. When a station does not receive an ACK within a predetermined timeout, it will double its $C W$ value and re-enter the backoff process, attempting to resend the frame. Upon consecutive unsuccessful transmission attempts, $C W$ can be doubled up to a maximum value $C W_{\max }=2^{m}\left(C W_{\min }+1\right)-1$, where $m$ denotes the maximum backoff stage. If further attempts fail, $C W$ is maintained at the maximum value and eventually the frame is discarded after a consecutive retry limit $K$ is exceeded. Otherwise, if the transmission is successful, the $C W$ is reset to $C W_{\min }$. The values of the involved parameters are summarised in Table 1 for the HR/DSSS (802.11b) physical layer [23].

\subsection{System Assumptions}

We consider a WLAN where nodes are within carrier sense range of one another and do not employ the RTS/CTS mechanism, as previous studies show this 4-way handshake procedure provides limited benefits in the absence of hidden terminals [24], while if used in combination with power control, it can harm throughput performance [25]. Our analysis, however, could be easily extended also to this case. We assume ideal channel conditions, therefore losses are only caused by collisions. Without loss of generality, we assume stations send fixed size packets and employ a single PHY rate. These assumptions are commonly used in the literature [1-4] and could be relaxed, but we expect that they do not significantly change our conclusions.

We analyse the 802.11 MAC behaviour with heterogeneous capture, distinguishing

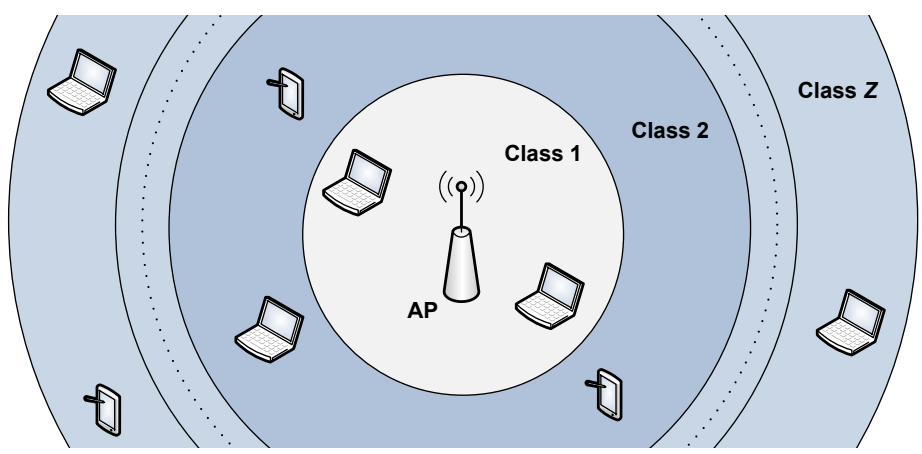

Fig. 1. Classes of stations with heterogeneous capture probabilities. 
$Z$ classes of nodes that experience dissimilar capture probabilities as a result of their placement relative to the AP. We index the classes according to their likelihood of capturing, as depicted in Fig. 1, i.e. the lower the class index, the closer a node within that class is to the AP and the more plausible it will be to capture when transmitting simultaneously with nodes of classes with higher indexes.

As such, nodes within any Class $j$ can capture the channel over nodes in Class $l$, $\forall l>j$. Consequently, when concurrent transmissions occur, the following outcomes are possible:

- If a station in Class $j$ and respectively a node in Class $l, l>j$, transmit $\Rightarrow$ station in Class $j$ captures with probability $\alpha_{j, l}$; transmission of station in Class $l$ fails;

- If a station in Class $j$ transmits simultaneously with any other station in Class $l$, $l \leq j, \Rightarrow$ transmission fails.

We treat the capture probabilities $\alpha_{j, l}$ as model parameters, while methods given in e.g. $[6,7]$ can be employed to accurately estimate their values. In what follows, we study the 802.11 throughput performance under different network loads in the presence of capture, to identify those scenarios in which all classes of stations could benefit from this effect.

\section{Performance Analysis of 802.11 with Heterogeneous Capture}

To study the performance of a WLAN where stations experience dissimilar capture probabilities, we first present a throughput model that accounts for this effect and then employ numerical analysis to illustrate that gains can be achieved in such circumstances, depending on the volume of traffic nodes generate. By investigating the relationship between the success probabilities, transmission attempt rates and arrival processes, we provide valuable insights into the observed behaviour.

\subsection{Throughput Model}

We propose a realistic model of 802.11 operation that, unlike previous works, allows nodes with heterogeneous capture probabilities. In the absence of the capture effect, simultaneous frame transmissions fail due to collisions. In this case, the conditional failure (collision) probability $p$ experienced by a transmitted frame is given by [1]:

$$
p=1-(1-\tau)^{n-1},
$$

where $\tau$ is the stationary probability that a station transmits in a randomly chosen slot time and $n$ is the number of stations in the WLAN. 
In the presence of the capture effect, a transmission of a station can be successful even when another station transmits simultaneously. However, only the frame received with a higher signal level will be decoded by the AP, while the other transmission will result in failure. Denoting by $p_{j}$ the conditional failure probability of a Class $j$ and by $\tau_{j}$ the corresponding transmission probability of stations in that class, we can relate the probability of success of a station in Class $j$ with the transmission probabilities of the other classes as follows:

$$
\begin{aligned}
1-p_{j}= & \left(1-\tau_{j}\right)^{n_{j}-1} \prod_{l=1, l \neq j}^{Z}\left(1-\tau_{l}\right)^{n_{l}} \\
& +\left(1-\tau_{j}\right)^{n_{j}-1} \sum_{l=j+1}^{Z}\left(1-\left(1-\tau_{l}\right)^{n_{l}}\right) \alpha_{j, l}\left(\prod_{w=1, w \neq j}^{l-1}\left(1-\tau_{w}\right)^{n_{w}}\right)
\end{aligned}
$$

where, as explained earlier, $\alpha_{j, l}$ is the probability that a station from Class $j$ captures the channel over stations from Class $l$, while $n_{j}, j \in\{1, . ., Z\}$, denotes the number of stations belonging to Class $j$. As in [12], we treat a fraction of the collisions as resulting in capture, instead of simply subtracting a capture probability from the collision probability to compute the failure probability, which is the approach previously used by e.g. $[5,8]$.

Next, we use a renewal-reward approach to analyse the 802.11 binary exponential backoff scheme. The advantage of using this approach is that it does not require the direct calculation of the stationary distribution of the Markov Chain [3,26], but instead we calculate the expected number of slots and transmissions between the renewal events, i.e. the completion of packet transmissions. Let $p$ be a station's conditional failure probability. The expected number of attempts to transmit a packet is

$$
E(R)=1+p+p^{2}+\ldots+p^{K},
$$

where $K$ is the maximum number of retry attempts. The expected number of slots used during backoff is

$$
E(X)=t_{i}+b_{0}+p b_{1}+p^{2} b_{2}+\ldots+p^{k} b_{k}+\ldots+p^{K} b_{K}
$$

where $b_{k}$ is the mean length of backoff stage $k$ expressed in slots and $t_{i}$ is the mean idle time that a station waits for a packet after transmission. Thus, we can express the transmission attempt rate of the station as

$$
\tau=\frac{E(R)}{E(X)}=\frac{1+p+p^{2}+\ldots+p^{K}}{t_{i}+b_{0}+p b_{1}+p^{2} b_{2}+\ldots+p^{K} b_{K}} .
$$

We apply the above equation to the considered classes of stations to relate each $\tau_{j}$ to the corresponding $p_{j}$. 
Neglecting post-backoff and assuming no buffering, we can write

$$
t_{i}=q\left(1+2(1-q)+3(1-q)^{2}+\ldots\right)=\frac{1}{q},
$$

where $q$ is the probability that a new frame arrives in a uniform slot time $E_{s}$, given by

$$
E_{s}=P_{i} \sigma+P_{s} T_{s}+P_{f} T_{f},
$$

in which $\sigma, T_{s}$ and $T_{f}$ are the average durations of an idle slot, a successful transmission and a failure respectively, and $P_{i}, P_{s}$ and $P_{f}$ are the corresponding probabilities, given by

$$
\begin{aligned}
P_{i} & =\prod_{j=1}^{Z}\left(1-\tau_{j}\right)^{n_{j}}, \\
P_{s} & =\sum_{j=1}^{Z} n_{j} \tau_{j}\left(1-p_{j}\right), \\
P_{f} & =1-P_{i}-P_{s},
\end{aligned}
$$

$T_{s}$ and $T_{f}$ can be expressed as

$$
\begin{gathered}
T_{s}=T_{P L C P}+\frac{E[L]}{C}+S I F S+T_{A C K}+D I F S, \\
T_{f}=T_{P L C P}+\frac{E[L]}{C}+T_{A C K_{\text {_timeout }},}
\end{gathered}
$$

where $T_{P L C P}$ is the duration of the PLCP (Physical Layer Convergence Protocol) preamble and header, ${ }^{1} L$ is the average frame length, $T_{A C K}$ is the duration of an acknowledgment, $C$ is the PHY rate and $T_{A C K \text { timeout }}$ is a physical layer constant.

Given $C W_{\max }=2^{m}\left(C W_{\min }+1\right)-1$ and using $W=C W_{\min }+1$ to simplify notation, we have $b_{k}=2^{k} W / 2, \forall k \geq 0$ and, assuming infinite backoff $(K \rightarrow \infty)$, we obtain:

$$
\tau_{j}=\frac{2\left(1-2 p_{j}\right)}{W\left(1-p_{j}-p_{j}\left(2 p_{j}\right)^{m}\right)+\frac{2\left(1-2 p_{j}\right)\left(1-p_{j}\right)}{q}} .
$$

Combining (2) and (11), we can solve $\left(\tau_{1}, p_{1}, \ldots, \tau_{Z}, p_{Z}\right)$ and compute the total throughput of the network as follows:

$$
S=\frac{P_{s} E[P]}{E_{s}}
$$

$\overline{1}$ E.g. 72 bit PLCP preamble transmitted at $1 \mathrm{Mb} / \mathrm{s}$ and 48 bit PLCP header transmitted at $2 \mathrm{Mb} / \mathrm{s}$, for HR/DSSS (802.11b) PHY layer with short preamble [23]. 


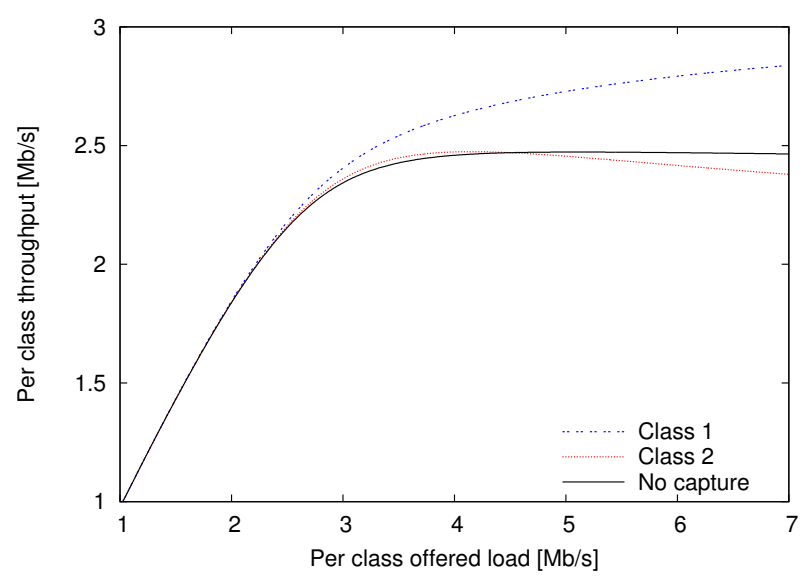

Fig. 2. Throughput performance with and without the capture effect.

where $E[P]$ is the expected size of the payload. The above completes our throughput model. Next, we study the impact of the offered load on the performance attained with the capture effect.

\subsection{Numerical Results and Discussion}

Using the proposed model, we consider a simple example scenario of a network with two classes of stations, each class yielding $n_{j}=5$ nodes, and analyse numerically the throughput attained by each class as the offered load is varied. ${ }^{2}$ We assume stations employ the 802.11 b system parameters, as summarised in Table 1, and are transmitting packets of fixed size, $E[P]=500$ bytes. We consider identical load at each node and $\alpha_{1,2}=0.75$, i.e. a Class 1 station can capture the channel over Class 2 stations $75 \%$ of the time. ${ }^{3}$ Note that the plots we present are obtained by numerically evaluating the model in Sec. 4.1, using the above network parameters along with a fine set of offered load values, and computing the expected performance.

The results are shown in Fig. 2, where we also plot for comparison the throughput performance of an identical network setup but without the capture effect (i.e. $\alpha=$ 0 ). As expected, the throughput of Class 1 is always above the throughput achieved by the same group of stations in a capture-free scenario. However, what is more interesting is that the throughput of Class 2 can also be larger in the presence of capture, depending on the offered load of Class 1 stations. Note that although the observed differences may appear small, it is worth comprehending what drives these improvements, as we will show later that capture can be exploited when nodes

2 We relate the arrival rate per slot $q$ to the actual packet arrival rate per second $\lambda$, with $\lambda=-\log (1-q) / E_{s}$. Assuming all the traffic is independent and Poisson, the offered load is obtained as $\lambda E[P]$.

3 Numerical analyses conducted with different values of $\alpha_{1,2}$ yield similar results. 


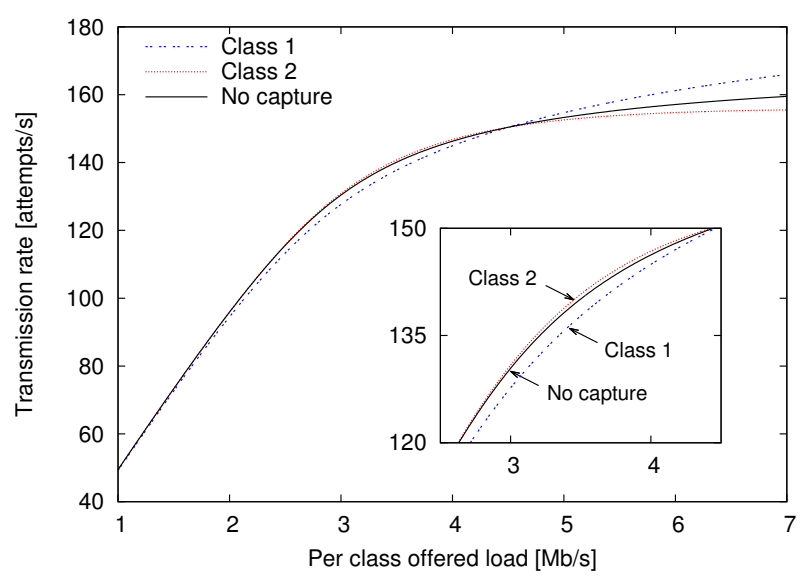

Fig. 3. Transmission rate, $\tau / E_{s}$.

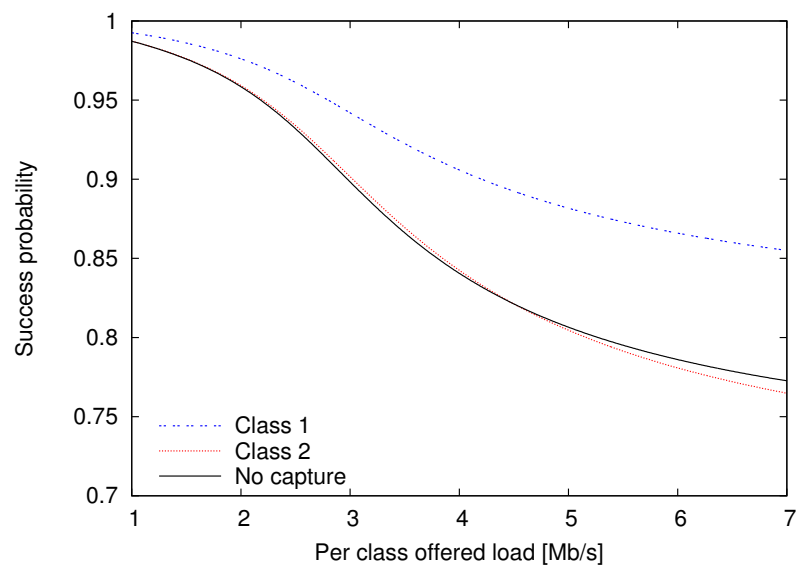

Fig. 4. Success probability, $(1-p)$.

experience similar channel conditions to enhance the overall network performance.

To better understand the perceived behaviour, let us first write the throughput for a single node in Class $j$ as:

$$
S_{j}=E[P] \frac{\tau_{j}}{E_{s}}\left(1-p_{j}\right) .
$$

Since $E[P]$ is fixed, the throughput variation can be explained by changes in the transmission rate, $\tau / E_{s}$, and in the success probability, $1-p$. Note that, because of the random access mechanism, these two quantities depend on one another, as already explained in Section 4.1. Therefore, to provide further insight into the evolution of the two terms, we separately plot these in Figs. 3 and 4, respectively, for the two classes of stations under study.

For the case of Class 1, Fig. 3 shows that the transmission rate can be smaller in the presence of capture if the network is lightly loaded. At the same time, Fig. 4 illustrates that the success probability seen by Class 1 is always higher than the one experienced by Class 2 in the network with capture. Thus, the overall throughput 
improvements of Class 1 are a nontrivial combination of tradeoffs between transmission rate and probability of success.

For the case of Class 2, similar tradeoffs occur. Namely, while the failure probability, $p$, increases with the offered load, the success probability, $1-p$, can actually become higher than in the equivalent network without capture, allowing the possibility of improved throughput over the capture-free case. At the same time, the transmission rate of Class 2 is equal or lower in the presence of capture as compared to the capture-free case. As the observed transmission rate effectively stops increasing at a low offered load while the failure probability continues to increase, this explains the peak in the throughput of Class 2 seen in Fig. 2.

To conclude this section, we will study the relationship between transmission and conditional failure probabilities. This will offer insight into how the throughput gains are related to differences in failure probability between the two classes and to the network load.

It is worth noting that, at very low offered loads, we observe that the transmission rate of Class 1 is lower than that of Class 2 . This may be initially regarded as surprising, since both classes have the same offered load and Class 2 sees a higher failure probability. In particular, as stations backoff upon failed attempts, higher failure probabilities are usually associated with lower transmission probabilities. Given this counter-intuitive behaviour, we further analyse the relationship between transmission and failure probabilities.

If we take the derivative of (5) with respect to $p$, we obtain

$$
\begin{aligned}
\frac{d \tau}{d p} & =\frac{\left(\sum_{k=0}^{K} b_{k} p^{k}+\frac{1}{q}\right)\left(\sum_{l=1}^{K} l p^{l-1}\right)}{\left(\sum_{k=0}^{K} b_{k} p^{k}+\frac{1}{q}\right)^{2}} \\
& -\frac{\left(\sum_{k=0}^{K} p^{k}\right)\left(\sum_{l=1}^{K} l b_{l} p^{l-1}\right)}{\left(\sum_{k=0}^{K} b_{k} p^{k}+\frac{1}{q}\right)^{2}} .
\end{aligned}
$$

Kumar et al. proved that in saturation (i.e. $q=1$ ), if $b_{l}>=b_{k}, \forall l>k$ and $b_{k}$ are not all equal, then $\tau$ is strictly decreasing with $p$ when $p \in(0,1)$ [3]. Therefore, the expression in (14) will be negative.

Conversely, when the network is lightly loaded, from our previous observation about the attempt rates of the two classes we conjecture that $\tau$ must not be decreasing. If we evaluate the numerator of (14) when the derivative changes sign, we can write:

$$
\begin{array}{r}
\left(p^{m}\left(4 m 2^{m} p+(-2 m-2) 2^{m}\right)+2\right) q^{2} W \\
+\left(8 p^{2}-8 p+2\right) q^{2}+\left(-8 p^{2}+8 p-2\right) q=0
\end{array}
$$




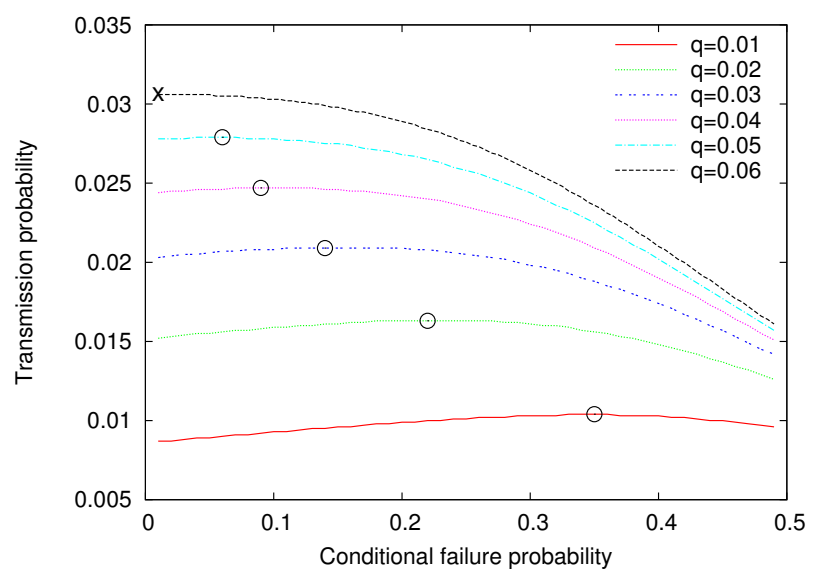

Fig. 5. Relationship between $\tau$ and $p$ for different arrival rates $q$. The circles indicate the maximum values of $\tau$.

In a lightly-loaded case (i.e. $p \approx 0$ ), the above is equivalent to:

$$
2 q^{2} W+2 q^{2}-2 q=0
$$

which has a root at $q_{0}=1 /(W+1)$. Intuitively, this tells us that when the arrival rate is smaller than one packet every $W+1$ slots, then increasing $p$ can increase $\tau$, due to the increased number of retransmission attempts.

In Fig. 5 we plot the relationship between $\tau$ and $p$ as given by (11) for different $q$ values. We observe that, for non-saturated scenarios, there exists a non-monotonic relationship between the transmission and collision probabilities, meaning an increase in $p$ can increase or decrease $\tau$. We also mark in the figure the turning point for each curve. To the left of this point increases in the failure probability result in increased transmission rate, and to the right increased failure probability results in decreased transmission rate. As $q$ increases, we see this point moves towards $p=0$. Following this observation, we could compute the $q$ value where the transmission rate becomes monotonic.

We conclude that, in the presence of the capture effect, the throughput increase arises from a tradeoff between the transmission and success probabilities, depending on the stations' offered load, and cannot be understood in terms of only one of the aforementioned quantities alone.

\section{Power-hopping MAC}

We have shown that in some circumstances the capture effect can boost the performance of all stations, as the increased success rate and the reduced number of retransmissions experienced by nodes near the AP yield an increase in the air time available for all contenders. In this section, we take our finding one step fur- 
ther and we conjecture that, in dense deployments prone to increased contention, by periodically setting the transmission power of stations to dissimilar levels, it would be possible to enhance the throughput performance of all stations. Therefore, we design a power-hopping MAC (PH-MAC) scheme that exploits the capture effect to mitigate collisions, thereby improving the performance of the basic 802.11 DCF mechanism. We quantify numerically the benefits of employing the proposed scheme and then present a prototype implementation. We conduct experiments with a real 802.11 deployment and demonstrate that PH-MAC provides significant performance gains without requiring changes to the existing hardware, but only some minor modification to the available open-source device drivers.

\subsection{PH-MAC Design}

Consider a situation where a station has determined that a power level $P_{\min }$ is sufficient to transmit reliably to the AP at the selected PHY rate and assume the maximum transmission power supported by the hardware is $P_{\max }$. Further, suppose that a station can select from $L$ discrete levels within a $\left[P_{\min }, P_{\max }\right]$ range and assume that the difference between any two distinct levels in the possible range is large enough to cause capture. Thus, when two stations transmit simultaneously using dissimilar power levels, the transmission at the higher level will capture with different probabilities over the lower power transmission, depending on their relative difference, and will be successfully decoded at the AP.

Following this observation, the objective of PH-MAC is to choose transmission powers so that a significant number of collisions result in capture. To this end, for each transmission attempt, PH-MAC randomly chooses a power level within the possible range. Specifically, we select among $L$ power levels with probabilities $\left\{p_{1}, p_{2}, \ldots, p_{L}\right\}$. We consider that stations use the same set of probabilities of choosing among distinct power levels. Thus, on average they experience similar capture probabilities and success rates. Further, our design is intended to use power levels above those where successful transmission has become dependable (see [27] for an experimental assessment of the relationship between packet loss rate and SNR).

The immediate question is how to properly configure these probabilities to optimise the network performance? To answer this, let us first model the expected behaviour of our proposal. Imagine a single cell 802.11 network with $n$ stations following the basic DCF rules and employing one of $L$ power levels at each transmission attempt, as explained above.

Initially, consider a symmetric network, where all stations have the same transmission rate $\tau$. Then, if all frames were transmitted with the same power level, the 
conditional collision probability $p_{c}$ could be written as

$$
p_{c}=1-(1-\tau)^{n-1}=\sum_{i=1}^{n-1}\left(\begin{array}{c}
n-1 \\
i
\end{array}\right) \tau^{i}(1-\tau)^{n-i-1} .
$$

However, since we employ the power-hopping scheme, some of the simultaneous transmissions will be successfully decoded because of capture, while others will still fail. Thus, the failure probability $p$ is given by

$$
\begin{aligned}
p= & \sum_{i=1}^{n-1}\left(\begin{array}{c}
n-1 \\
i
\end{array}\right) \tau^{i}(1-\tau)^{n-i-1}\left[p_{1}\left(1-\left(p_{2}+p_{3}+\ldots+p_{L}\right)^{i}\right)\right. \\
& \left.+p_{2}\left(1-\left(p_{3}+p_{4}+\ldots+p_{L}\right)^{i}\right)+\ldots+p_{L-1}\left(1-p_{L}^{i}\right)+p_{L}\right],
\end{aligned}
$$

which can be reduced to

$$
p=\sum_{i=1}^{n-1}\left(\begin{array}{c}
n-1 \\
i
\end{array}\right) \tau^{i}(1-\tau)^{n-i-1} \sum_{l=1}^{L} p_{l}\left(1-\left(\sum_{k=l+1}^{L} p_{k}\right)^{i}\right) .
$$

Given that in practice $\tau$ takes small values (i.e. $\tau \ll 1$ ), the term corresponding to $i=1$ dominates the expression of the conditional failure probability given by (16). Thus we can approximate $p$ by

$$
p \approx p_{c}(\underbrace{1-\sum_{l=1}^{L-1} p_{l} \sum_{k=l+1}^{L} p_{k}}_{\text {no_cap }}) .
$$

Examining the above, we reason that, in a case where failures are dominated by collisions of two stations, the failure probability will be scaled by the term denoted no_cap relative to the collision probability $p_{c}$ (of the capture-free case). Thus, in order to optimise performance we need to find the set of $\left\{p_{1}, \ldots p_{L}\right\}$ probabilities that minimise the failure probability, which is equivalent to solving the following optimisation problem:

$$
\begin{array}{ll} 
& \min _{\left\{p_{1}, \ldots, p_{L}\right\}} \text { no_cap } \\
\text { s.t. } & \sum_{l=1}^{L} p_{l}=1, \\
& 0 \leq p_{l} \geq 1, l=1, \ldots, L .
\end{array}
$$

The corresponding Lagrangian is given by

$$
L(p, \lambda)=n o \_c a p+\lambda\left(\sum_{l=1}^{L} p_{l}-1\right)
$$




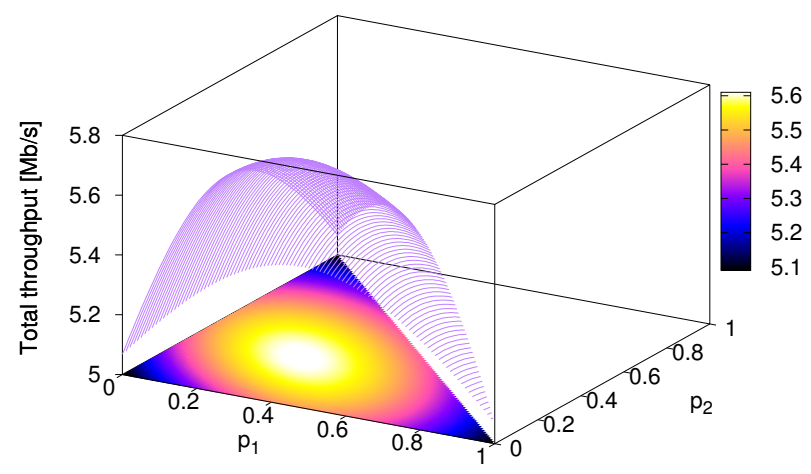

Fig. 6. Throughput performance with power-hopping using different $p_{h}$ values, under saturation conditions.

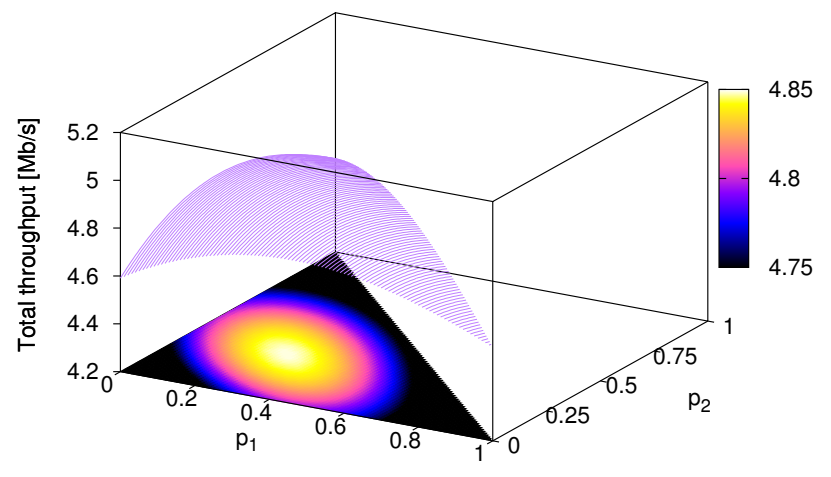

Fig. 7. Throughput performance with power-hopping using different $p_{h}$ values, under non-saturation conditions.

and the solution is obtained by setting the gradient of $L(p, \lambda)$ to zero, i.e.

$$
\frac{\partial}{\partial p_{j}}\left(1-\sum_{l=1}^{L-1} p_{l} \sum_{k=l+1}^{L} p_{k}+\lambda\left(\sum_{l=1}^{L} p_{l}-1\right)\right)=0, j=1, . ., L
$$

which after solving yields

$$
p_{j}=\lambda-1, \forall j
$$

The above means that the failure probability is minimised when all power levels are randomly chosen with equal probability, i.e.

$$
p_{j}=\frac{1}{L}, j=1, \ldots, L .
$$

To validate this result, we use (11) to solve numerically (16) and apply (12) to predict the throughput performance of the protocol for different values of the $p_{j}, j=$ $1, \ldots, L$ probabilities. Through this method, we plot the throughput of a saturated 
and non-saturated network with $n=10$ stations in Figs. 6 and 7 respectively, for the possible range of $p_{j}$ values, considering nodes choose from $L=3$ power levels. Clearly, in both cases, choosing $p_{j}=1 / 3, \forall j$ provides noticeable performance benefits as compared to the capture-free scenario $\left(p_{j}=1\right.$ and $\left.p_{k}=0, \forall k \neq j\right)$.

\subsection{Numerical Results}

To estimate the benefits of using the proposed scheme, we first investigate numerically the throughput performance of PH-MAC as compared to the standard 802.11 MAC, varying the network conditions in terms of numbers of active stations and considering different offered loads.

We start by examining a network with saturated stations, sending again 500 byte packets and employing the $802.11 \mathrm{~b}$ parameters (see Table 1), and evaluate the throughput attained by PH-MAC and basic DCF as the number of active stations increases. At the same time, we study the impact the number of power levels $L$ used by PH-MAC has on the network throughput. As shown in Fig. 8, by randomly choosing among the available power levels on each transmission, PH-MAC outperforms DCF, the throughput gain increasing with $n$. In particular, observe that PH-MAC alleviates much of the loss due to collisions in networks with more than five stations. Notice as well that employing $L>3$ power levels does not provide significant benefits over the $L=3$ setting, which is somewhat expected as in practice employing more levels would reduce the differences among the received signal strength of the transmitted packets and consequently the capture probability upon simultaneous transmissions.

To understand the impact of the offered load on the performance of the proposed enhancement, we analyse a scenario in which 10 nodes are present in the WLAN and we consider different packet arrival rates. The results are illustrated in Fig. 9. We

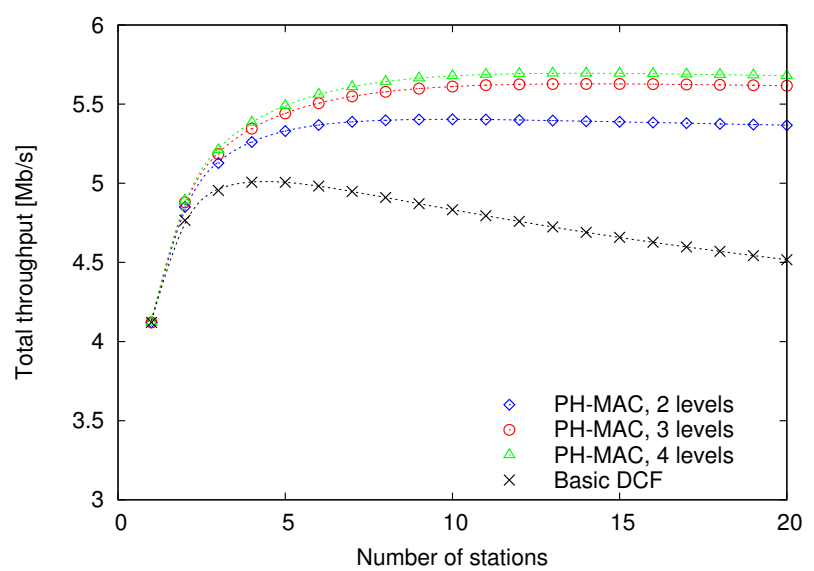

Fig. 8. Throughput performance of PH-MAC compared with basic DCF, varying the network size, under saturation conditions. 


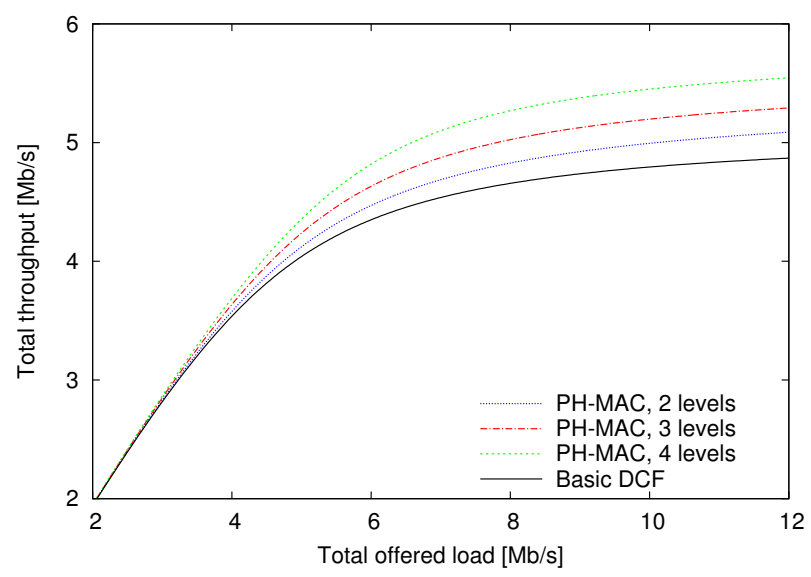

Fig. 9. Throughput performance of PH-MAC compared with basic DCF, varying the offered load.

observe that, as the offered load increases, PH-MAC provides increasing throughput gains over the basic DCF mechanism. Note that, as we model stations with small buffers, the behaviour depicted in Fig. 9 corresponds to a system that has not reached saturation (i.e. $q<1$ ), hence the plotted throughput has not yet reached the value for $n=10$ stations shown in Fig. 8 .

Following these results, we conclude that PH-MAC successfully exploits the capture effect to improve the performance of dense networks. These findings motivate a practical assessment of PH-MAC in real environments. To this end, we next present a prototype implementation, which we experimentally evaluate in a smallscale 802.11 testbed.

\subsection{Prototype Implementation}

We argue that the power-hopping enhancement we introduced can be effectively implemented with existing hardware, only with relatively basic driver modifications. To support our claim, in what follows we describe a prototype implementation we developed using the popular open-source MadWifi v0.9.4 driver. ${ }^{4}$ Note that due to the inherent limitations of the driver, which we discuss next, our prototype is an approximation of the proposed PH-MAC scheme. However, as our experimental results will show, the implemented prototype can significantly outperform the standard DCF in real deployments.

The key advantage of using MadWifi drivers with Atheros-based wireless cards over other practical 802.11 solutions is that they support Transmit Power Control (TPC) functionality, i.e. they allow setting the transmission power level with a perpacket granularity, as already reported in $[28,29]$. In the PH-MAC approach, so far

$\overline{4 \text { http }}: / /$ madwifi-project.org 
we have assumed that the power can be changed with each transmission attempt. However, this would require a fine control of the hardware, namely accessing the retransmission-handling routines, that are generally embedded in the hardware abstraction layer (HAL) or firmware. Unfortunately, the source code implementing this low-level functionality is not publicly available for the existing devices. Consequently, our prototype only tunes the transmission power on a per-packet instead of per-transmission attempt basis. Nonetheless, we expect only minor deviations from the predicted performance and we will demonstrate that, indeed, our implementation achieves noteworthy throughput gains despite this approximation.

To implement PH-MAC we modified the part of the driver's source code handling the transmission operations for the Atheros wireless LAN controller (if_ath). Precisely, when a new packet arrives at the MAC queue, an ath_tx_start routine is invoked to handle the transmission. After performing the encapsulation operations, computing the transmission duration and selecting the antenna to be used for transmission, the routine prepares a transmission descriptor that is used to pass the packet to the hardware, along with a set of PHY layer parameters, among which is the transmission power. To achieve the desired power-hopping functionality, before the transmission descriptor is provided to the hardware through the ath_hal_setuptxdesc call, we update the transmission power parameter retained in the node information structure (ni), that is passed at the call, and invoke the ath_update_txpow routine, that sets the TX power of the device to the desired level.

Although a predefined set of power levels can be configured, to ensure a significant difference between the power levels used for transmissions is achieved and a high likelihood of capture exists even under small deployments, we opt for implementing PH-MAC with $L=2$ levels, thus randomly choosing between a low and a high power with probability $p_{1}=p_{2}=0.5$. To this end, when enqueueing a packet, we generate a random unsigned 32-bit number using the random 32 routine, which we compare to the the value that splits the possible range in half, i.e. $0 \times 7 F F F F F F F$. The power level passed for update has to be expressed as $0.5 \mathrm{dBm}$ increments, e.g. for setting the power to $P_{\max }=16 \mathrm{dBm}$ a value of 32 is expected. ${ }^{5}$ Note that MAC operation as specified by the standard is kept unmodified. For further details about the implementation, we refer the reader to the source code of the prototype, which we make publicly available online. ${ }^{6}$ In what follows we validate PH-MAC's operation in an 802.11 testbed.

\footnotetext{
5 The maximum power level that can be set on a device varies with manufacturers and is subject to regulatory constraints.

6 http://www.hamilton.ie/ppatras/\#code
} 


\subsection{Experimental Evaluation}

To evaluate the potential performance benefits of PH-MAC in a real environment, we conduct experiments in a small testbed that we deployed at the Hamilton Institute in Ireland. The network is placed in a dynamic office space with numerous occupants and the experiments are conducted during working hours. Thus the radio conditions are typical of an active environment with humans. The testbed consists of 7 nodes: one PC acting as access point and 6 Soekris net 4801 embedded PCs serving as clients. All nodes are equipped with Wistron CM9 Atheros miniPCI $802.11 \mathrm{~b} / \mathrm{g}$ cards, $5 \mathrm{dBi}$ omnidirectional antennas, and run the Ubuntu 8.04 LTS Linux distribution with kernel 2.6.24. The WLAN is operating on channel 6 $(2.437 \mathrm{GHz})$ where no other networks have been detected and thus we conclude it is an interference free environment. RTS/CTS, turbo, fast frame, bursting and unscheduled automatic power save functionalities are disabled in all experiments, while the antenna diversity scheme is not employed for transmission/reception. All nodes are within LOS from the AP, thus we expect negligible multipath propagation effects.

Given the reduced size of our deployment we configure nodes with $C W_{\min }=7$ and $C W_{\max }=15$, to ensure an increased level of contention in the WLAN. ${ }^{7}$ Thus we seek to replicate conditions typical of practical scenarios that are subject to moderate-to-high loads in order to study the performance in circumstances DCF finds challenging, such as conference halls, libraries, auditoriums, etc. Also, because of the compactness of our testbed, we are free to select between the lowest and highest configurable powers upon transmission, i.e. $0 \mathrm{dBm}(1 \mathrm{~mW})$ and $16 \mathrm{dBm}$ $(40 \mathrm{~mW})$. This ensures a high likelihood of capture when transmissions at different levels overlap. Given the limited capabilities of the stations $(266 \mathrm{MHz} \mathrm{CPU}$ and $128 \mathrm{MB}$ physical memory), we configure their PHY rate to $11 \mathrm{Mb} / \mathrm{s}$ (CCK). We expect, however, that our findings will hold also for the OFDM PHY, as capture occurs for all non-linear modulation-based 802.11 PHY layer technologies [10]. In all tests, we disable rate adaptation, but recognize that capture probability could change depending on the PHY rate in use. ${ }^{8}$ We expect similar qualitative results in such scenarios. With these settings, we proceed with comparing the performance of PH-MAC and basic DCF under different network conditions in terms of link quality, application type and traffic loads.

\footnotetext{
7 Note that these are realistic MAC settings, as they are employed for e.g. the video queue of the $802.11 \mathrm{a} / \mathrm{g}$ modes.

8 We refer the reader to [10] for a detailed discussion on the relationship between PHY rate and SIR threshold for capture.
} 
Table 2

RSSI footprint of the testbed with nodes transmitting at $16 \mathrm{dBm}$.

\begin{tabular}{|c|c|c||c|c|}
\hline \multicolumn{4}{c}{ Homogeneous environment } & Heterogeneous environment \\
\hline $\begin{array}{c}\text { Station index } \\
\text { index }\end{array}$ & $\begin{array}{c}\text { RSSI average } \\
{[\mathrm{dBm}]}\end{array}$ & $\begin{array}{c}\text { RSSI std. dev. } \\
{[\mathrm{dB}]}\end{array}$ & $\begin{array}{c}\text { RSSI average } \\
{[\mathrm{dBm}]}\end{array}$ & $\begin{array}{c}\text { RSSI std. dev. } \\
{[\mathrm{dB}]}\end{array}$ \\
\hline 1 & -39.4 & 4.2 & -51.6 & 4.0 \\
2 & -39.5 & 4.5 & -38.0 & 5.9 \\
3 & -38.8 & 3.6 & -41.0 & 3.9 \\
4 & -38.4 & 4.1 & -47.3 & 4.2 \\
5 & -38.4 & 3.7 & -50.4 & 3.5 \\
6 & -39.4 & 3.8 & -40.6 & 4.8 \\
\hline Avg. min. & \multicolumn{3}{|c|}{-39.5} & \multicolumn{3}{c|}{-51.6} \\
Avg. max. & -38.4 & 38.0 \\
\hline
\end{tabular}

\subsubsection{Homogeneous Environment}

We first consider a homogeneous environment, whereby clients experience similar link qualities to the AP. For this purpose, we position the nodes at comparable distances from the AP to guarantee frames are received with similar signal strength when transmitted at the same power level. To confirm this, we periodically send ICMP traffic between each station and the AP, and record the received signal strength indicators (RSSI) for the received frames as reported in the radiotap header of the packets sniffed with t cpdump. Specifically, nodes send sequentially 10 probe requests, with 1 second spacing in between, and packet size of 1000 bytes. After each round, stations pause for 1 minute and then repeat this process 5 times, to capture link quality variations due to external factors such as human interference. In Table 2, on the left side, we provide the average and standard deviation of the RSSI for each node sending at maximum TX power. We observe that, indeed, all nodes experience almost identical link qualities when sending to the AP.

Following these preliminary measurements, we investigate the performance gains of PH-MAC when stations send UDP traffic to the AP. To this end, we use the iperf tool to generate UDP packets with 500 byte payload, uniformly varying the offered load at each node and recording the total throughput at the AP, first when clients employ PH-MAC, alternating between the minimum and maximum power levels for transmissions, and then with the basic DCF mechanism in place. Each experiment runs for 3 minutes and is repeated 10 times, to obtain average values of the throughput with good statistical significance.

The results are shown in Fig. 10, where we plot average and 95\% confidence intervals of the throughput obtained with the two approaches. As observed in the figure, 


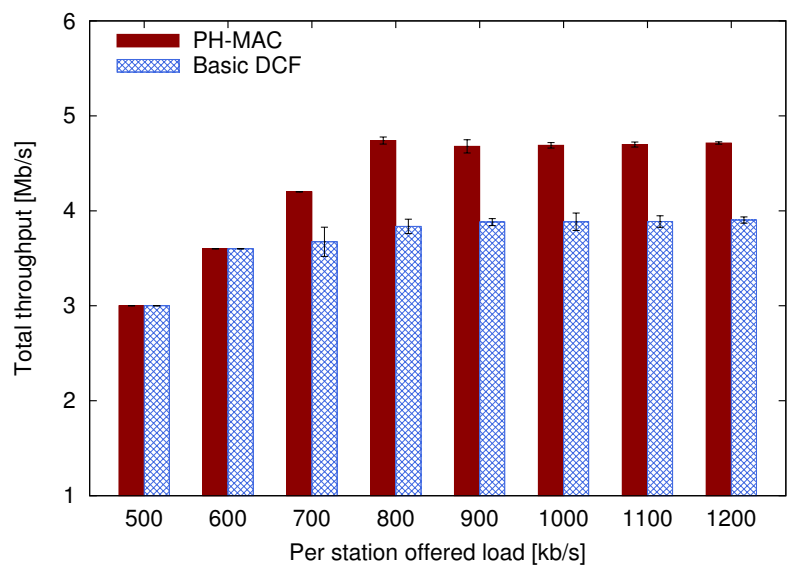

Fig. 10. Experimental evaluation of PH-MAC under similar link conditions.

PH-MAC significantly outperforms the basic DCF mechanism as stations generate more traffic, showing notable benefits as the network approaches saturation $(\lambda \geq$ $800 \mathrm{~kb} / \mathrm{s}$ ). In particular, by changing between low and high transmission power levels, PH-MAC achieves throughput gains of up to $25 \%$ over the standard scheme.

\subsubsection{Heterogeneous Environment}

Next, we study the impact of heterogeneous link qualities on the performance of PH-MAC, to understand whether performance gains are achieved also under such conditions. Besides potential throughput benefits, here we also seek to investigate whether our approach is able to improve fairness among stations in scenarios where nodes closer to the AP would have by default a higher likelihood of capture and potentially obtain more throughput than nodes far away. To obtain such a topology, we randomly place the stations at different distances relative to the AP. We then repeat the procedure used in Section 5.4.1 to determine the RSSI footprint of the deployment. We give the average and standard deviation of the RSSI of the links from each station to the AP in the right columns of Table 2. The values confirm that, indeed, in this scenario clients experience dissimilar links. ${ }^{9}$

Once again, we generate UDP traffic from the clients to the AP, using packets with 500 byte payload, varying the offered load and repeating the experiments 10 times to achieve good accuracy. We measure the individual throughput performance attained by each station and compute the total throughput and the network utility as the sum of the logs of the individual throughputs. The latter has been shown to be a relevant indicator of achieving a good compromise between optimising the network capacity and providing competing nodes with a good level of fairness [30]. More specifically, for the case of the 802.11 protocol, the higher the utility becomes, the

$\overline{9}$ The difference between the minimum and maximum average link qualities is $\approx 14 \mathrm{~dB}$, which represents a twenty-five times difference in signal strength. Thus we conclude this corresponds to a significant degree of heterogeneity. 


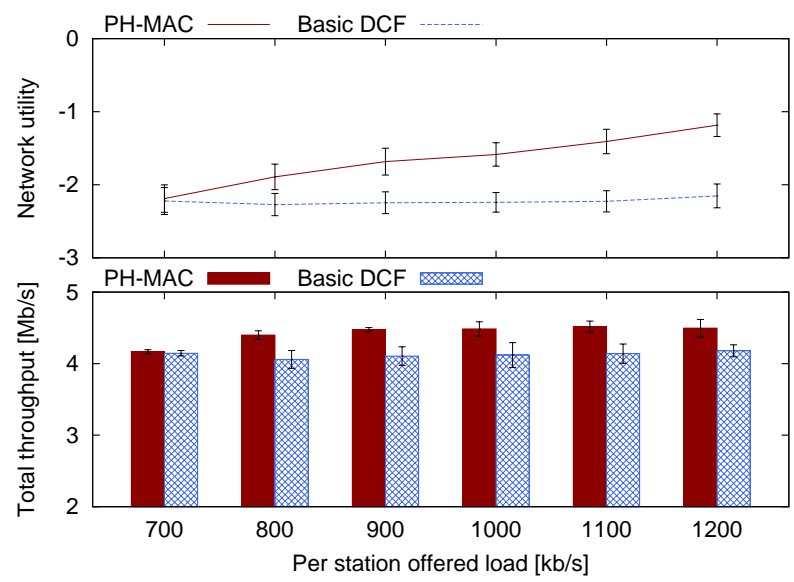

Fig. 11. Experimental evaluation of PH-MAC under dissimilar link conditions.

closer the stations get to obtaining equal air-time [31]. We plot the two metrics as a function of the offered load in Fig. 11. As results show, PH-MAC not only provides higher throughput as compared to the basic DCF mechanism, but also helps restoring fairness in heterogeneous links environments, increasing network utility.

\subsubsection{TCP File Transfers}

We further investigate the performance of PH-MAC under the previous scenario with heterogeneous link qualities, this time considering a network with bidirectional traffic. Rather than fully saturating the network with synthetic traffic, our goal here is to understand the benefits of our proposal under representative web workloads, that mimic the behaviour of real users. Specifically, stations establish finite size TCP connections, alternating between periods of activity, during which a 10 Mbyte file is transferred, and silent periods exponentially distributed with mean $\lambda^{-1}=60 \mathrm{~s}$ [32]. We run such experiments for a total time of 3 hours with both the basic DCF mechanism and the proposed PH-MAC scheme, logging all transfer durations and computing the per-station average delay. We expect dissimilar link conditions in combinations with existing congestion avoidance mechanisms in current TCP implementations to exacerbate unfairness. Thus our second goal here is to understand whether PH-MAC can improve fairness also with such traffic. To illustrate the distribution of the average delay among nodes with the two approaches, we use a box-and-whisker plot.

As depicted in Fig. 12, when employing PH-MAC stations attain reduced transfer delays as compared to the basic DCF, as the median (marked with dark line) is approximately $10 \mathrm{~s}$ lower with our approach (42 s with PH-MAC, compared to $52 \mathrm{~s}$ with basic DCF). Further, the distance between the first and third quartiles is smaller with PH-MAC, which shows that most of the stations experience similar performance. This contrasts to the significantly different delays nodes attain with the basic DCF due to dissimilar link qualities. 


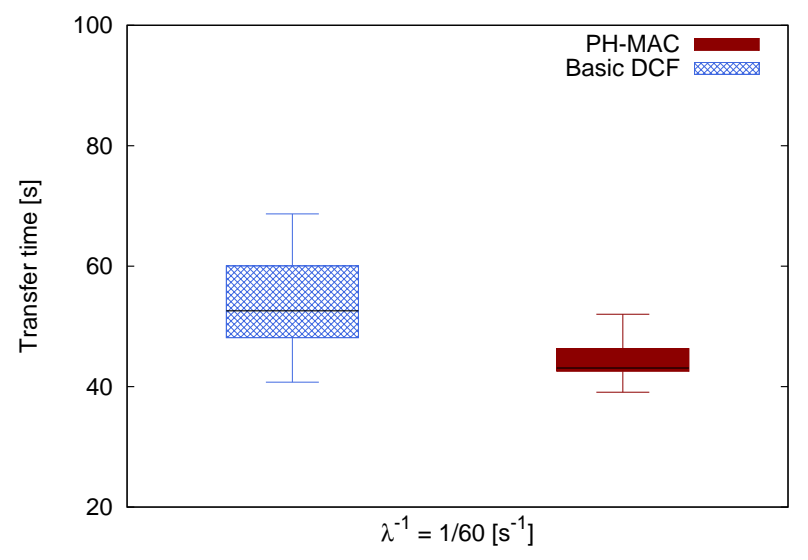

Fig. 12. Experimental evaluation of PH-MAC with bidirectional traffic.

We conclude that, by alternating between a low and a high power level, our PHMAC implementation is able to provide noteworthy throughput gains over the default DCF scheme, both in homogeneous and heterogeneous radio environments, while improving fairness and transfer delay in topologies prone to uneven performance due to the relative placement of the clients with respect to the AP.

\section{Conclusions}

In this paper, we analysed the performance of the 802.11 protocol with heterogeneous capture probabilities, and showed that the capture effect can enhance the performance of all nodes in the networks, depending on the load conditions. Based on this analysis, we introduced a power-hopping technique, $\mathrm{PH}-\mathrm{MAC}$, that selects different power levels upon transmission, thus purposely causing capture to improve performance in dense deployments. We implemented a practical approximation of PH-MAC, which we evaluated in a real 802.11 testbed under different conditions in terms of link qualities, network loads and traffic types. The obtained results show that PH-MAC can achieve noteworthy performance gains over the basic DCF scheme, providing up to $25 \%$ more throughput, increasing network utility, considerably improving fairness and reducing delay.

\section{References}

[1] G. Bianchi, Performance analysis of the IEEE 802.11 distributed coordination function, IEEE Journal on Selected Areas in Communications (JSAC) 18 (3) (2000) $535-547$.

[2] H. Wu, Y. Peng, K. Long, S. Cheng, J. Ma, Performance of reliable transport protocol over IEEE 802.11 wireless LAN: analysis and enhancement, in: Proc. IEEE 
INFOCOM, 2002, pp. 599-607.

[3] A. Kumar, E. Altman, D. Miorandi, G. Munish, New insights from a fixed-point analysis of single cell IEEE 802.11 WLANs, IEEE/ACM Trans. Netw. 15 (2007) 588601.

[4] D. Malone, K. Duffy, D. Leith, Modeling the 802.11 distributed coordination function in nonsaturated heterogeneous conditions, IEEE/ACM Trans. Netw. 15 (1) (2007) $159-172$.

[5] X. Ge, D. Yan, Y. Zhu, Throughput model of IEEE 802.11 networks with capture effect, in: Proc. IEEE WiCOM, 2006, pp. 1-4.

[6] Z. Hadzi-Velkov, B. Spasenovski, Capture effect in IEEE 802.11 basic service area under influence of Rayleigh fading and near/far effect, in: Proc. IEEE PIMRC, Vol. 1, 2002, pp. 172-176.

[7] Z. Hadzi-Velkov, B. Spasenovski, On the capacity of IEEE 802.11 DCF with capture in multipath-faded channels, International Journal of Wireless Information Networks 9 (2002) 191-199.

[8] G. Sutton, R. Liu, X. Yang, I. Collings, Modelling capture effect for 802.11 DCF under Rayleigh fading, in: Proc. IEEE ICCC, 2010.

[9] A. Kochut, A. Vasan, A. Vasan, A. U. Shankar, A. Agrawala, Sniffing out the correct physical layer capture model in 802.11b, 2004, pp. 252-261.

[10] J. Lee, W. Kim, S.-J. Lee, D. Jo, J. Ryu, T. Kwon, Y. Choi, An experimental study on the capture effect in 802.11a networks, in: Proc. ACM WinTECH, Montreal, Quebec, Canada, 2007, pp. 19-26.

[11] N. Santhapuri, R. R. Choudhury, J. Manweiler, S. Nelakuduti, S. Sen, K. Munagala, Message in Message MIM: A case for reordering transmissions in wireless networks, in: Proc. HotNets, 2008.

[12] P. Patras, H. Qi, D. Malone, Exploiting the Capture Effect to Improve WLAN Throughput, in: IEEE International Symposium on a World of Wireless, Mobile and Multimedia Networks (WoWMoM), 2012.

[13] C. Lau, C. Leung, Capture models for mobile packet radio networks, in: Proc. IEEE ICC, Vol. 30, 1990, pp. 1226-1230.

[14] M. Zorzi, R. Rao, Capture and retransmission control in mobile radio, IEEE Journal on Selected Areas in Communications (JSAC) 12 (8) (1994) 1289-1298.

[15] P. Serrano, A. Banchs, P. Patras, A. Azcorra, Optimal configuration of 802.11e EDCA for real-time and data traffic, IEEE Transactions on Vehicular Technology 59 (5) (2010) 2511-2528.

[16] K. Kosek-Szott, M. Natkaniec, A. R. Pach, A simple but accurate throughput model for IEEE 802.11 EDCA in saturation and non-saturation conditions, Comput. Netw. 55 (3) (2011) 622-635. 
[17] F. Peng, K. Shafiee, V. C. M. Leung, Throughput Modeling of Differentiation Schemes for IEEE 802.11e MAC Protocol, in: Proc. IEEE VTC Fall, 2012, pp. 1-5.

[18] S. Ganu, K. Ramachandran, M. Gruteser, I. Seskar, J. Deng, Methods for restoring MAC layer fairness in IEEE 802.11 networks with physical layer capture, in: Proc. REALMAN, Florence, Italy, 2006, pp. 7-14.

[19] J. Manweiler, N. Santhapuri, S. Sen, R. Roy Choudhury, S. Nelakuditi, K. Munagala, Order matters: Transmission reordering in wireless networks, IEEE/ACM Transactions on Networking 20 (2) (2012) 353 -366.

[20] D. Qiao, S. Choi, A. Jain, K. Shin, Adaptive transmit power control in IEEE 802.11a wireless LANs, in: Proc. VTC Spring, Vol. 1, 2003.

[21] V. P. Mhatre, K. Papagiannaki, F. Baccelli, Interference Mitigation through Power Control in High Density 802.11 WLANs, in: Proc. IEEE INFOCOM, 2007.

[22] K. Ramachandran, R. Kokku, H. Zhang, M. Gruteser, Symphony: Synchronous TwoPhase Rate and Power Control in 802.11 WLANs, IEEE/ACM Transactions on Networking 18 (4) (2010) 1289-1302.

[23] IEEE 802.11 WG, Wireless LAN Medium Access Control (MAC) and Physical Layer (PHY) specifications, IEEE Std 802.11, 2007.

[24] I. Tinnirello, S. Choi, Y. Kim, Revisit of RTS/CTS exchange in high-speed IEEE 802.11 networks, in: Proc. IEEE WoWMoM, 2005, pp. 240-248.

[25] I. Broustis, J. Eriksson, S. V. Krishnamurthy, M. Faloutsos, Implications of power control in wireless networks: A quantitative study, in: Proc. Passive and Active Measurement Conference (PAM), 2007.

[26] K. Duffy, Mean field Markov models of wireless local area networks, Markov Processes and Related Fields 16 (2) (2010) -.

[27] K. Huang, K. R. Duffy, D. Malone, H-RCA: 802.11 collision-aware rate control, IEEE/ACM Trans. Netw. (2012) - .

[28] K. Kowalik, M. Bykowski, B. Keegan, M. Davis, Practical issues of power control in IEEE 802.11 wireless devices, in: Proc. IEEE ICT, 2008, pp. 1-5.

[29] V. Shrivastava, D. Agrawal, A. Mishra, S. Banerjee, T. Nadeem, Understanding the limitations of transmit power control for indoor WLANS, in: Proc. ACM IMC, San Diego, USA, 2007.

[30] F. Kelly, Charging and rate control for elastic traffic, European Transactions on Telecommunications 8 (1) (1997) 33-37.

[31] A. Checco, D. Leith, Proportional Fairness in 802.11 Wireless LANs, IEEE Comms Letters 15 (8) (2011) 807-809.

[32] P. Barford, M. Crovella, Generating representative web workloads for network and server performance evaluation, in: Proc. ACM SIGMETRICS, Madison, United States, 1998, pp. 151-160. 